Kamil Jóźwik, Michał Ceremuga and Adam Tchórzewski

\title{
1. INDIVIDUAL PROTECTION EQUIPMENT
}

This section presents a basic overview of modern personal respiratory and skin protection equipment.

\subsection{Personal respiratory protection equipment}

Depending on the task in hand, the following types of respiratory protection equipment are available:

- Cleaning equipment - for protection from harmful substances in the air (dusts, gases, vapours etc);

- Isolation equipment - for oxygen-deficient atmospheres $\left(-17 \% \mathrm{O}_{2}\right)$;

- Escape equipment.

\subsubsection{Cleaning equipment}

Cleaning equipment purifies contaminated air of gases, aerosols, dust and chemicals. It does this using:

- Filters - to stop particulates (dust) and aerosols;

- Absorbers - to suspend chemicals in a gaseous state;

- Combined filters - with both filtering and absorbing actions.

Filters, absorbers and combined filters are used in various types of masks and respirators, which come in two varieties: half-masks (Fig. 1) and full-face masks (Fig. 2 and 3).

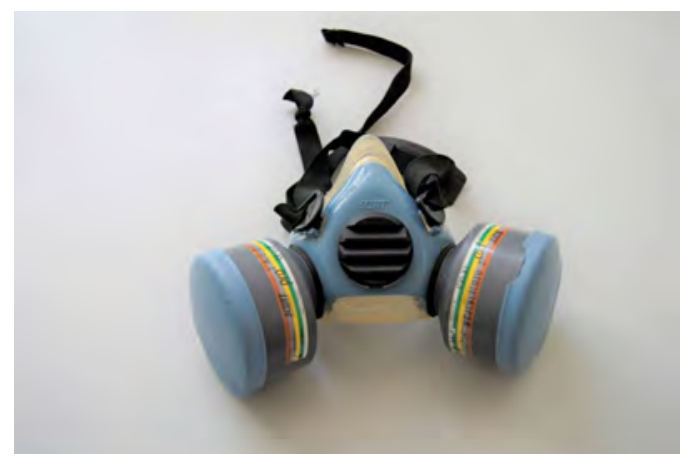

Figure 1. Half-mask with combined ABEKP filters (author's photo) 


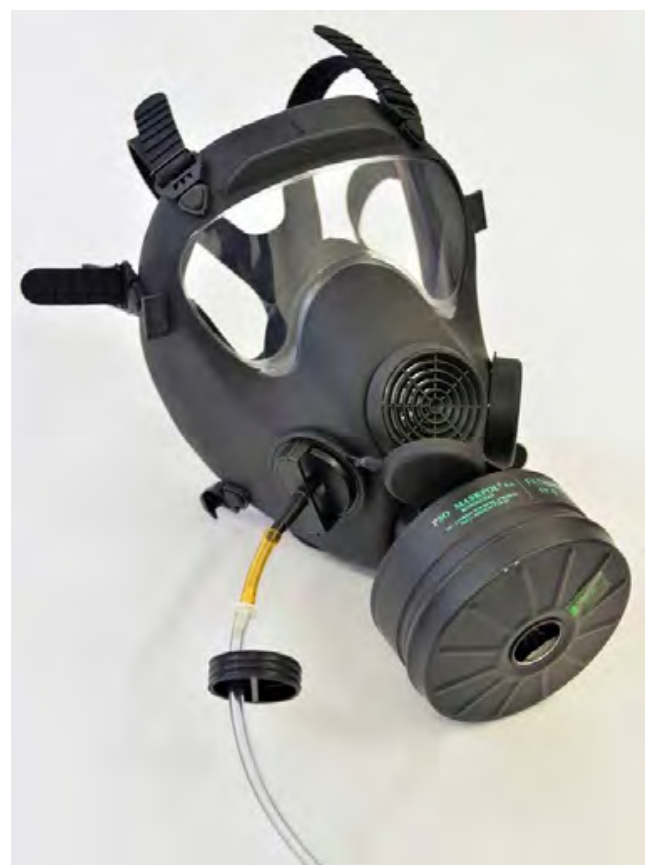

Figure 2. MP-5 gas mask used by the Polish army (author's photo)

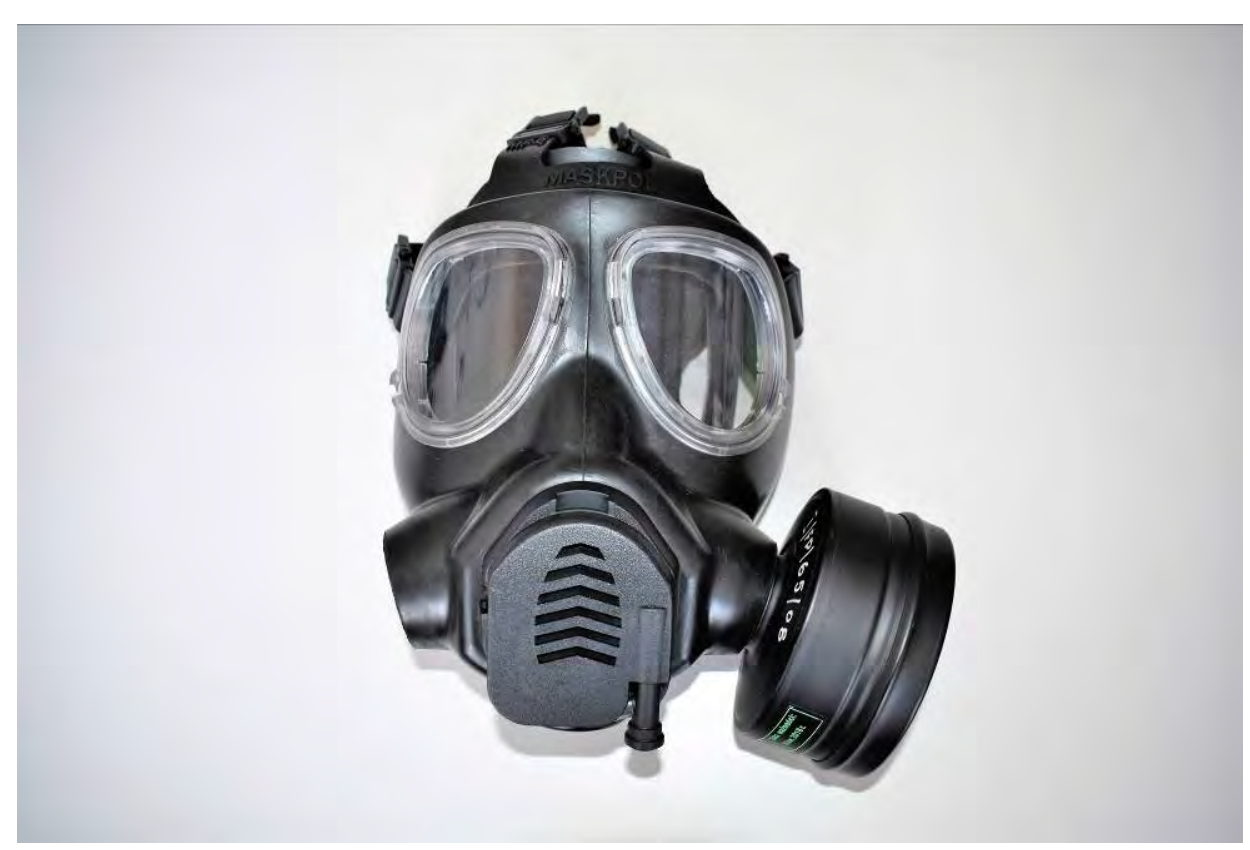

Figure 3. MP-6 gas mask used by the Polish army (author's photo) 
Depending on need, absorbers and combined filters are further divided into various types, as shown in Table 1 below.

Table 1. The different types of mask filters, with the symbols and colour codes used to designate their intended use

\begin{tabular}{|l|l|l|}
\hline Type & Colour & \multicolumn{1}{c|}{ Main application } \\
\hline $\mathrm{P}$ & white & Dust, particles \\
\hline $\mathrm{A}$ & brown & Gases and vapours of organic compounds with boiling point $>65^{\circ} \mathrm{C}$ \\
\hline $\mathrm{AX}$ & brown & Gases and vapours of organic compounds with boiling point $\leq 65^{\circ} \mathrm{C}$ \\
\hline $\mathrm{B}$ & grey & $\begin{array}{l}\text { Inorganic gases and vapours, e.g. chlorine, hydrogen sulfide, } \\
\text { hydrogen cyanide (excluding carbon dioxide/monoxide) }\end{array}$ \\
\hline $\mathrm{E}$ & yellow & Sulphur dioxide and other acidic vapours and gases \\
\hline $\mathrm{K}$ & green & Ammonia and ammonia derivatives and gases \\
\hline $\mathrm{Hg}$ & red & Mercury vapours \\
\hline
\end{tabular}

Figures $4-8$ show the markings used by absorber and filter manufacturers:

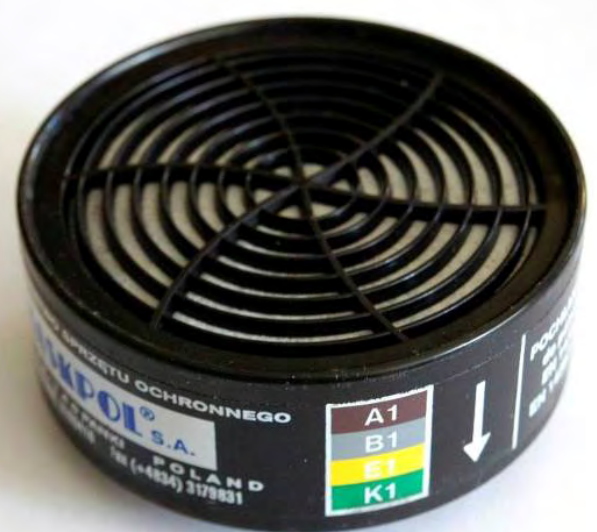

Figure 4. A1B1E1K1 Absorber - Type ABEK, Class 1 (author's photo) 


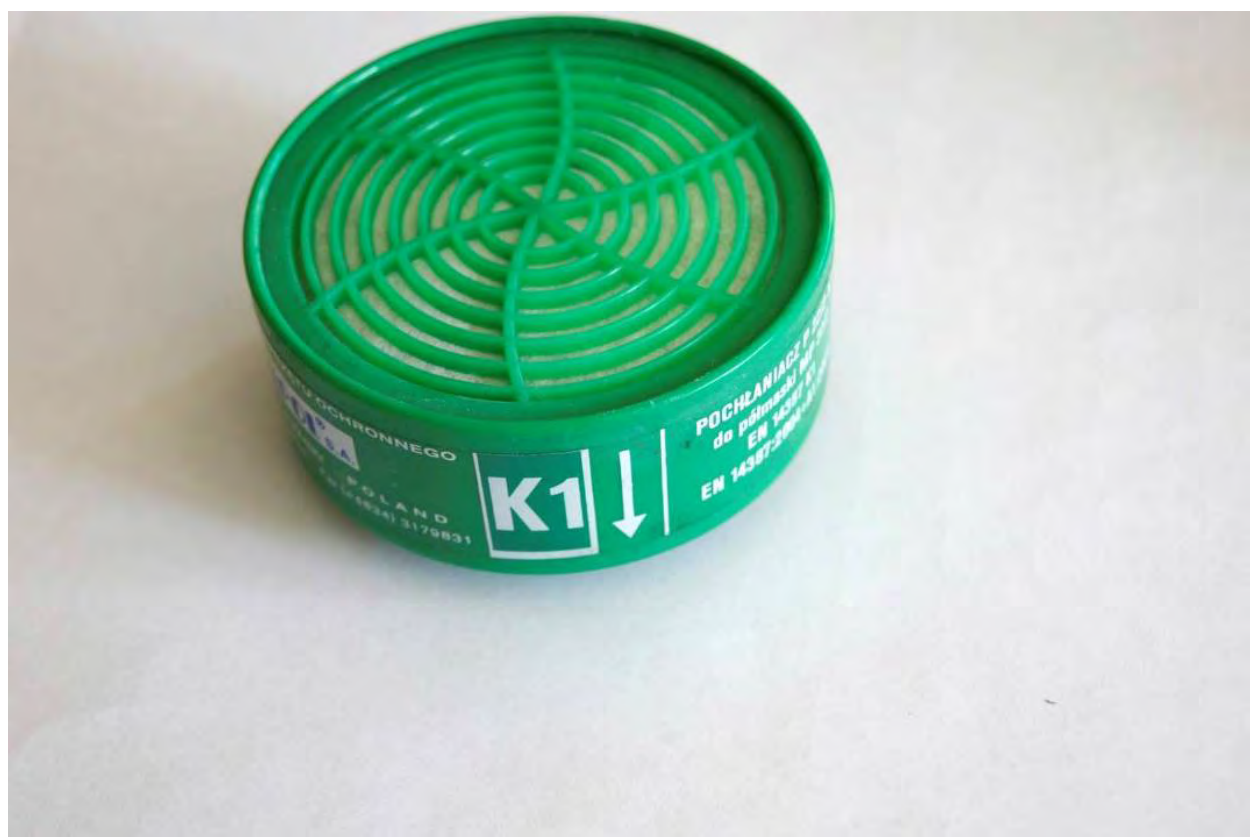

Figure 5. K1 Absorber - Type K, Class 1 (author's photo)

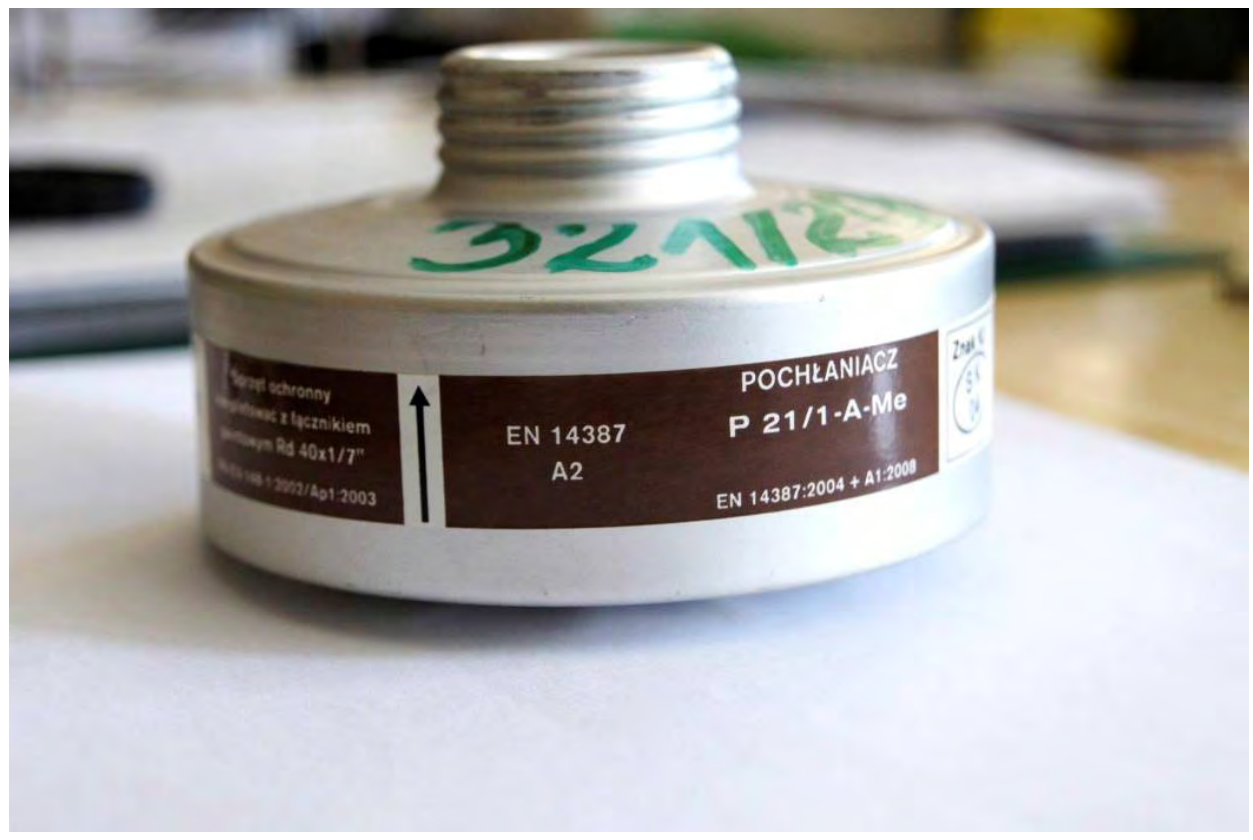

Figure 6. A2 Absorber - Type A, Class 2 (author's photo) 


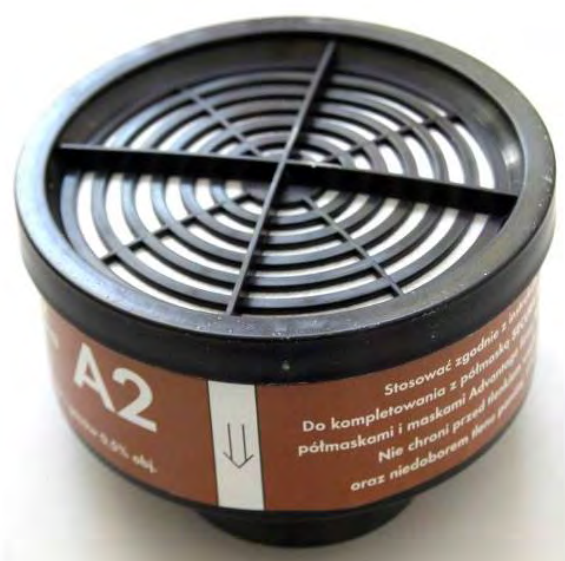

Figure 7. A2 Absorber - Type A, Class 2 (author's photo)

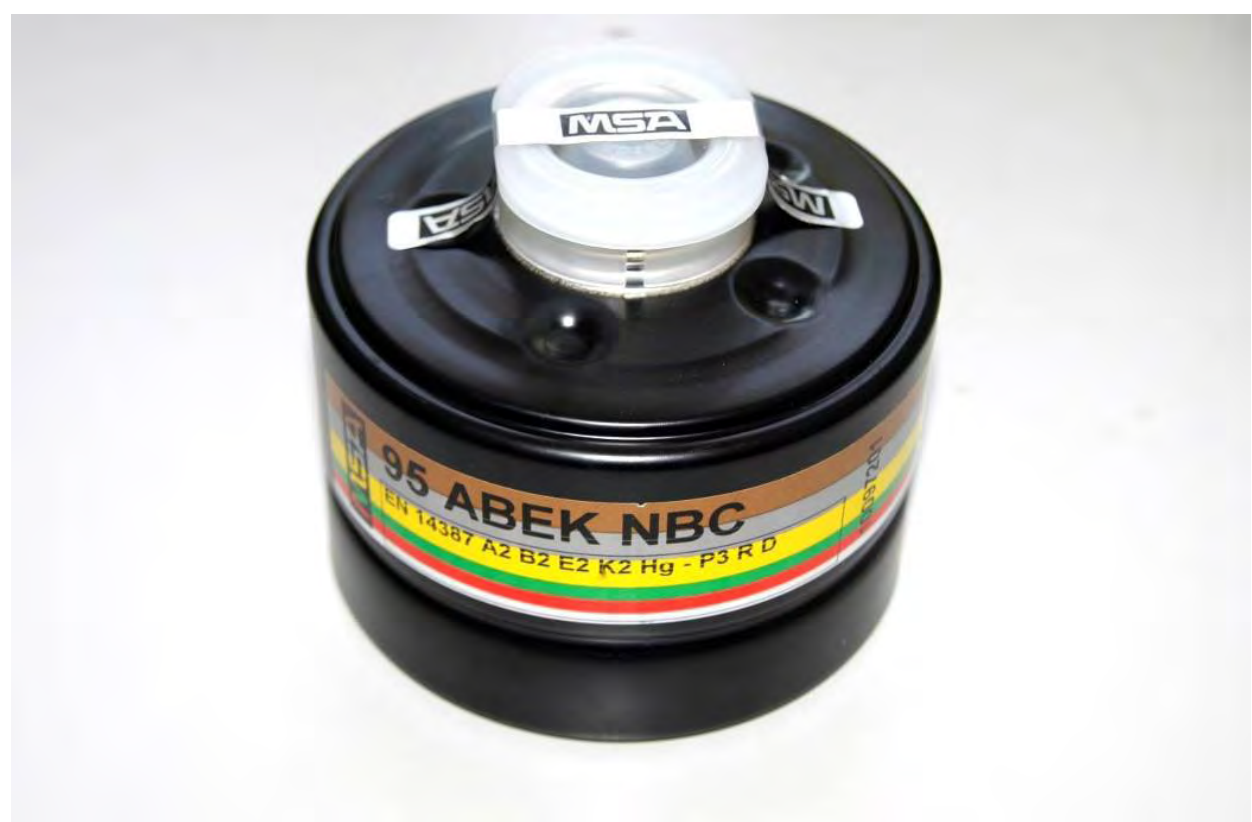

Figure 8. A2B2E2K2HgP3 combined filter - Type ABEKHg, Class 2 filter (P2) Class 3 (P3) (author's photo) 
Apart from categorising absorbers by the kinds of substances they protect against, they also have one of three class divisions:

- Class 1 - Low-absorbent sorbent, designed to protect against gases or vapours in airborne concentrations of up to $0.1 \%$;

- Class 2 - Medium sorption, designed to protect against gases or vapours with airborne concentrations of up to $0.5 \%$;

- Class 3 - Highly absorbent sorbent, designed to protect against gases or vapours in airborne concentrations of up to $1 \%$.

The type and class of each absorber of combined filter type determines its minimum protection time (Table 2 ).

Table 2. Minimum protection (breakthrough) time in minutes

\begin{tabular}{|c|l|c|c|}
\hline $\begin{array}{c}\text { Type, } \\
\text { class }\end{array}$ & \multicolumn{1}{|c|}{ Test substance } & $\begin{array}{c}\text { Minimum } \\
\text { breakthrough } \\
\text { time in minutes }\end{array}$ & $\begin{array}{c}\text { Concentration } \\
\text { of test substance } \\
\text { in air (\%) }\end{array}$ \\
\hline A1 & cyclohexane & 70 & 0.1 \\
\hline B1 & $\begin{array}{l}\text { Chlorine/hydrogen sulfide/ } \\
\text { hydrogen cyanide }\end{array}$ & $20 / 40 / 25$ & 0.1 \\
\hline E1 & Sulfur dioxide & 20 & 0.1 \\
\hline K1 & Ammonia & 50 & 0.1 \\
\hline A2 & cyclohexane & 35 & 0.5 \\
\hline B2 & $\begin{array}{l}\text { Chlorine/hydrogen sulfide/ } \\
\text { hydrogen cyanide }\end{array}$ & $20 / 40 / 25$ & 0.5 \\
\hline E2 & Sulfur dioxide & 20 & 0.5 \\
\hline K2 & Ammonia & 40 & 0.5 \\
\hline A3 & cyclohexane & 65 & 1.0 \\
\hline B3 & $\begin{array}{l}\text { Chlorine/hydrogen sulfide/ } \\
\text { hydrogen cyanide }\end{array}$ & $30 / 60 / 35$ & 1.0 \\
\hline E3 & Sulfur dioxide & 30 & 1.0 \\
\hline K3 & Ammonia & 60 & 1.0 \\
\hline
\end{tabular}

Filters are also classified by their efficiency of filtration, in their case against sodium chloride aerosols and paraffin oil mist (DEHS):

- Class 1 (P1) - filtration efficiency of $80 \%$. Protects against low toxicity solids for which the WEL long-term value is $\geq 2 \mathrm{mg} / \mathrm{m}^{3}$;

- Class 2 (P2) - filtration efficiency of $94 \%$. Protects against low and medium toxicity and solid particles for which the WEL long-term value is $\geq 0.05 \mathrm{mg} / \mathrm{m}^{3}$;

- Class 3 (P3) - filtration efficiency of $99.95 \%$. Protects against solids and liquids with high toxicity for which the WEL long-term value is $<0.05 \mathrm{mg} / \mathrm{m}^{3}$. 


\section{Filter half-masks}

Filters for half-mask respirators are also classified into one of three protection classes:

- Class 1 (FFP1) - filtration efficiency of $80 \%$. Protects against solids and liquids with low toxicity for which the WEL long-term value is $\geq 2 \mathrm{mg} / \mathrm{m}^{3}$ at maximum concentrations of $4 \mathrm{x}$ the WEL long-term value;

- Class 2 (FFP2) - filtration efficiency of 94\%. Protects against solids and liquid particles with low and medium toxicities for which the WEL long-term value is $0.05 \mathrm{mg} / \mathrm{m}^{3}$ at maximum concentrations of $10 \mathrm{x}$ the WEL long-term value;

- Class 3 (mark FFP3) - filtration efficiency of 97\%. Protects against solids and liquids with high toxicity, for which the WEL long-term value is $<0.05 \mathrm{mg} / \mathrm{m}^{3}$ at maximum concentrations of $20 \mathrm{x}$ the WEL long-term value.

- The following designations are used by the manufacturers:

- S - half mask designed for filtration of particulate matter (dust, smoke) only;

- SL - half mask designed for the filtration of particulates (dust, smoke) and liquid particles (aerosols, mist);

- D - additional requirement for dust absorption against dolomite dust;

- C - additional dust requirements for carbon dust.

\subsubsection{Isolation equipment}

Isolation suits provides a supply of clean air from an independent source (oxygen tanks), so that the user is completely cut off from contaminated air, even under reduced oxygen conditions. Isolation equipment is divided into:

- Stationary (with a compressed air hose and generator);

- Autonomous (with a tank or recycling system).

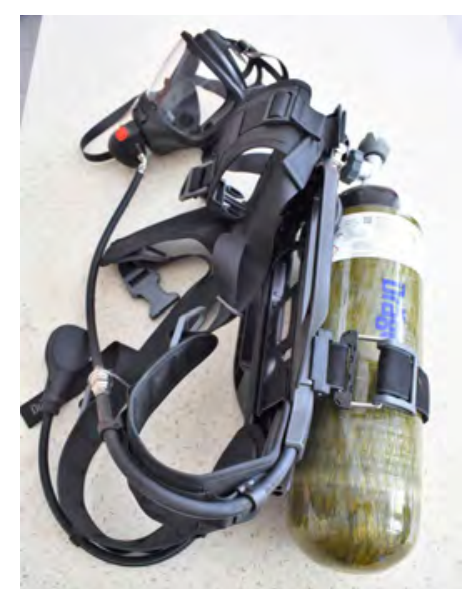

Figure 9. Isolation breathing apparatus (author's photo) 


\subsubsection{Escape equipment}

The third kind of protective respiratory gear is considered 'escape equipment', as it is intended not for prolonged working use, but simply for emergency use in the fast evacuation of hazardous areas. A good example of escape equipment is the protective hood and cover shown in figures 10 and 11, below.

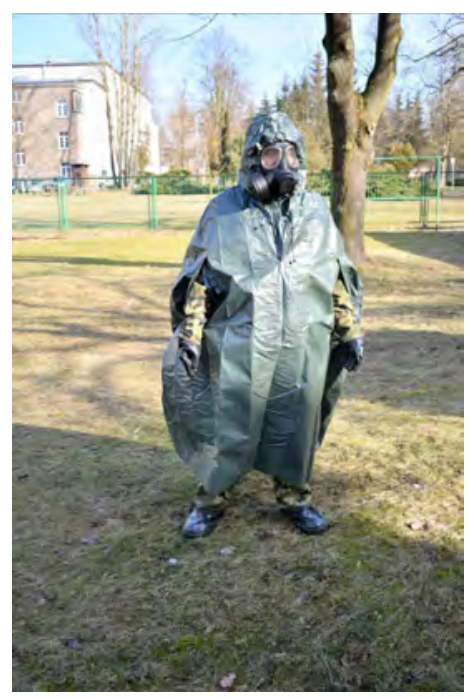

Figure 10. Protective cover (author's photo)

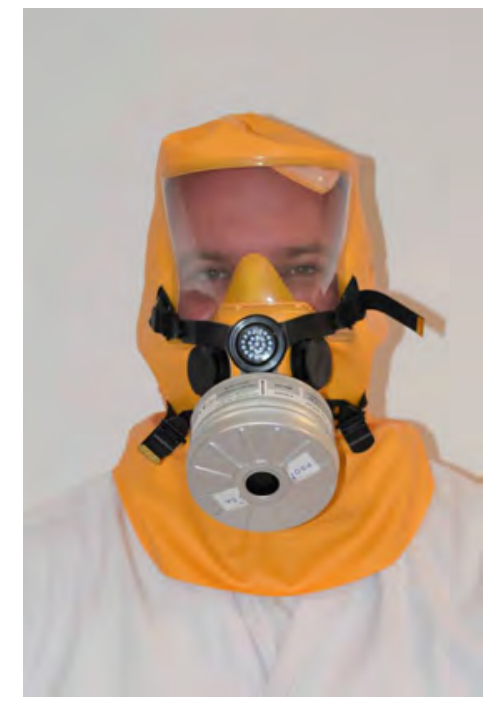

Figure 11. KO-1 protective hood (author's photo) 


\subsection{Personal skin protection equipment}

Skin protection equipment is used to protect the wearer's body surface from the harmful effects of poisonous substances. Based on the materials used and the purpose of the equipment, the protective layers of such clothing are designed to:

- Isolate harmful substances;

- Filter out harmful substances.

\subsubsection{Isolation suits}

As the name implies, isolation suits provide an impermeable barrier against contaminated environments. This type of clothing is usually worn by sub-units of chemical military troops, chemical disaster rescue teams, and medical and chemical rescue services. They provide excellent protection against dangerous chemicals (including Chemical Warfare Agents (CWAs)), and biological agents.

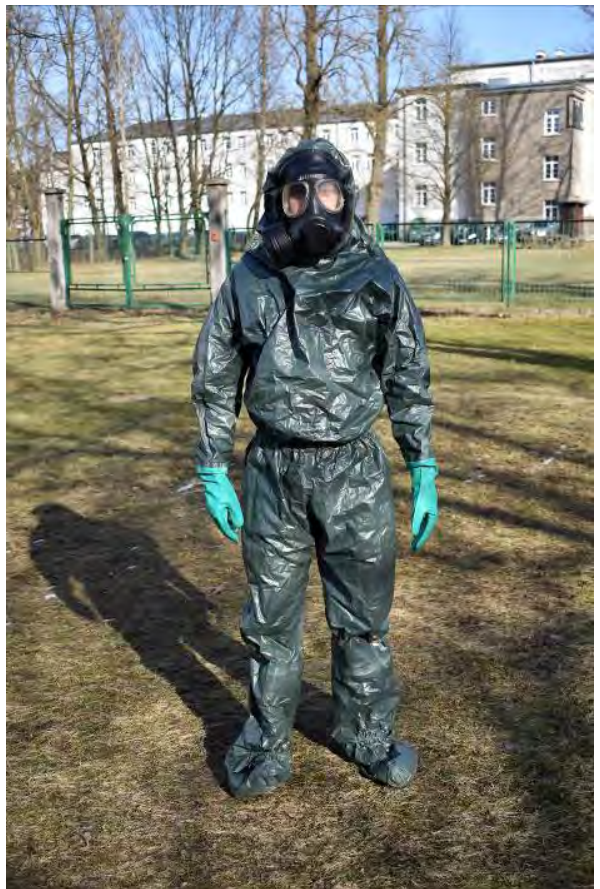

a)

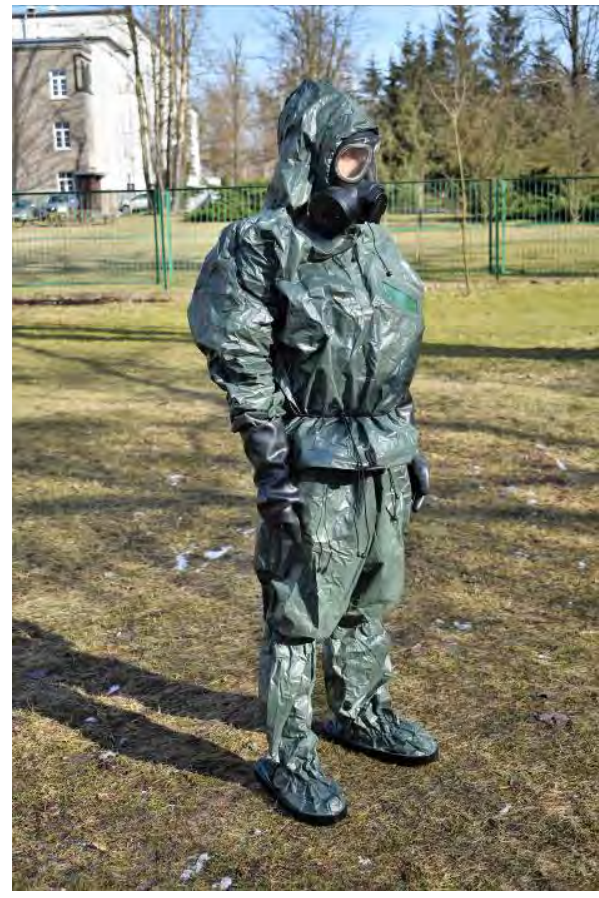

b)

Figure 12. Examples of lightweight clothing a) one-piece Tychem b) two-part LIO Maskpol (author's photo) 
Gas-tight suits are also used by specialised chemical rescue workers, and can take the form of one-piece suits with a gas-tight lock. They provide the highest level of protection and are designed for use in particularly hazardous conditions, such as with CWAs, Toxic Industrial Chemicals (TICs), and low oxygen levels. They are designed for use with breathing apparatus, and most importantly, can be decontaminated after use.

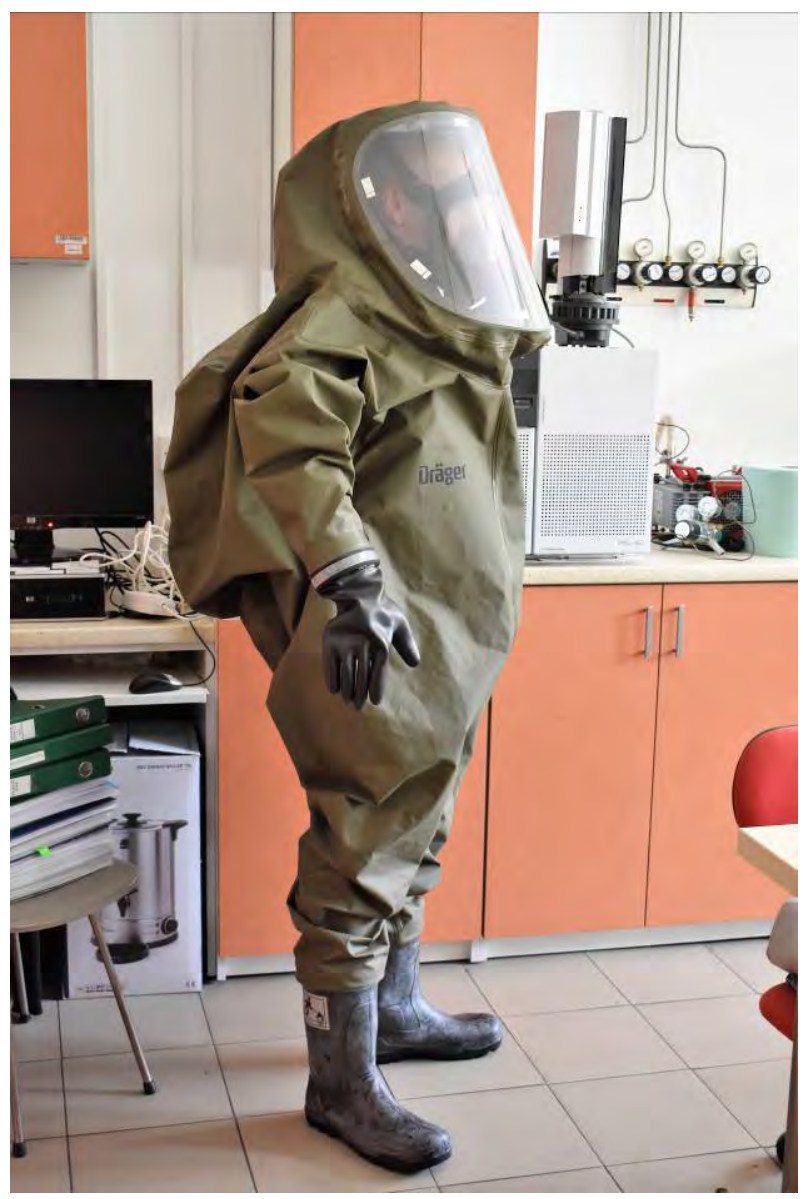

Figure 13. Examples of gas-tight suit type 1 (author's photo)

\subsubsection{Filter clothing}

Filter clothing is made with the use of absorbent materials. It protects against gaseous substances, but is not very effective against liquid substances (aerosols etc.). Filter clothing passes the wearer's heat and humidity (sweat) out, thus 
enhancing user comfort. Due to its low protection value against liquids, the shoes and gloves of protective filter clothing are made of isolating materials. Unlike isolation suits, filter clothing cannot be decontaminated after use.

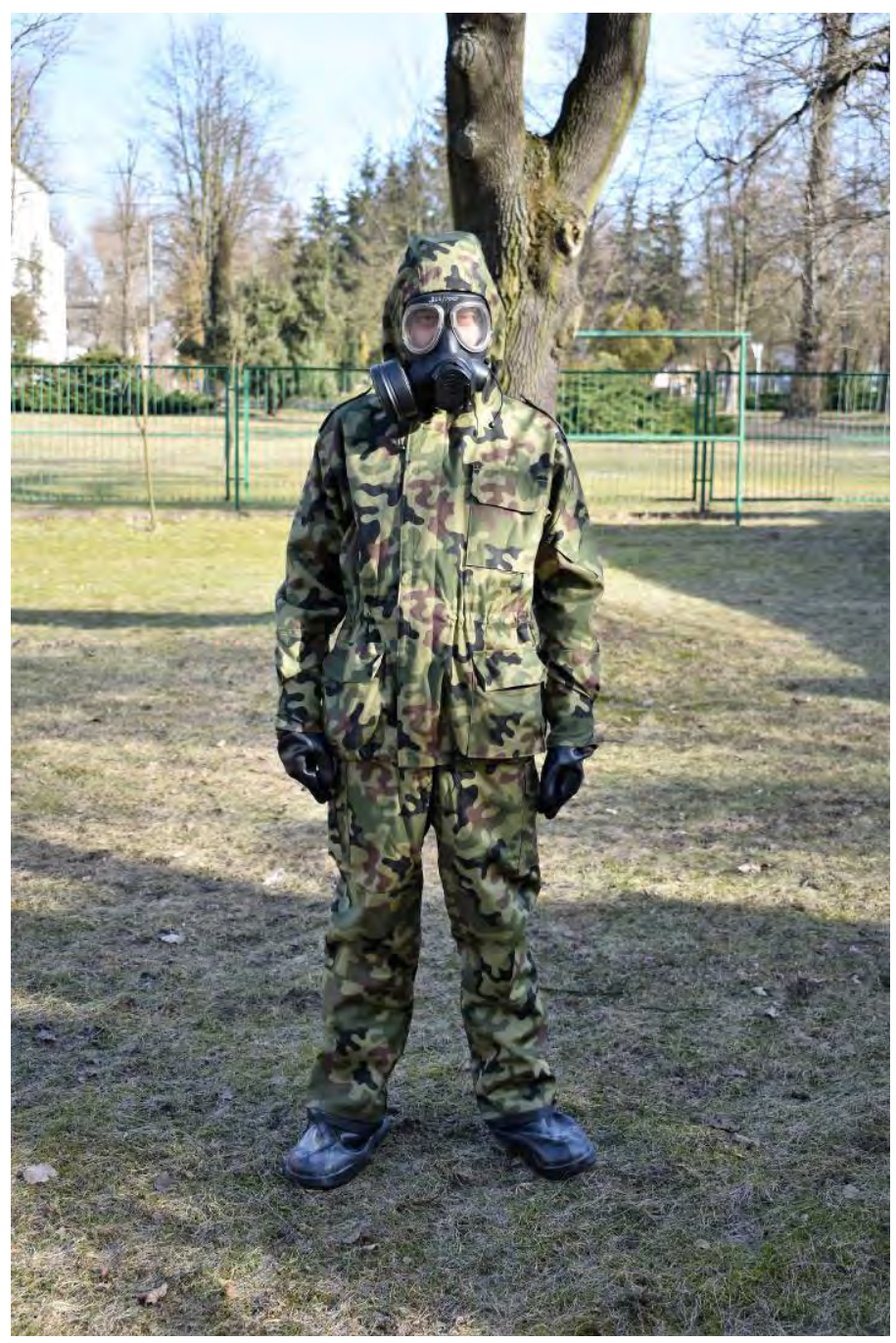

Figure 14. FOO-1 Filter Cloth (author's photo) 

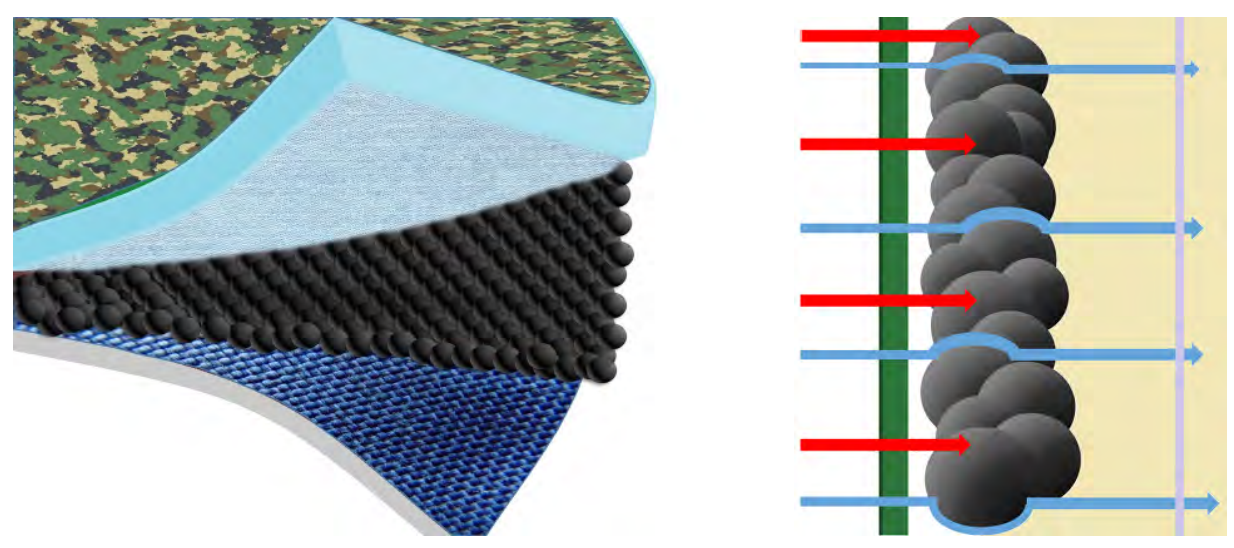

Figure 15. SARATOGA material with spherical, activated carbon

\subsection{Selection of appropriate protection equipment}

The following environmental conditions at an incident scene involving chemicals will determine the selection of appropriate personal protective equipment:

- Amount of oxygen in the air - If the oxygen content is below $17 \%$, use only isolation equipment (compressed air from a generator or from oxygen tanks). Keep in mind that the local oxygen content may also suddenly decrease, for example in confined spaces such as tanks, wells, ducts etc, or when oxygen could be displaced by another gas.

- Type of hazardous substance present - The first priority is to identify the harmful substances at the incident scene. If this cannot be determined, only isolation equipment can be used; do not use cleaning equipment.

- Type of contamination - Determine whether the hazardous substance(s) take the form of water-based mists or aerosols (use particle filters $(S)$ ); dispersed liquid aerosols (use particle and liquid filters (SL)); gas and steam (use absorbers); combinations of aerosols, vapours and gases (use combined filters).

- Concentration of the hazardous substance.

- Work intensity - The harder or more intensely the suit/mask-wearing operators are expected to work, the more clean air they will need to breathe.

- Working time - The usable time of respiratory protective equipment is sometimes limited by the specifications of the equipment itself. For example, oxygen tanks contain only a limited amount of air; filters can only filter so much hazardous material before they need replacing; very high concentrations of hazardous substances reduce the usable time of an absorber, and so on. When long working hours are expected, it is important to use high-quality equipment that is comfortable to use. Use of a system with an airflow should also be considered. 
- Ambient temperature, humidity - Elevated temperatures at an incident scene will lead to increased effort by the operators. This then leads to high humidity from sweating inside a full-face mask or suit, especially when combined with high-intensity work. Also, be aware that elevated temperatures and high humidity can shorten the duration of work of air suckers.

- Visibility - All types of respiratory protection reduce their wearer's field of vision. This is especially true of full-face masks, so use of those with the largest possible lenses is highly recommended.

- Communication - Masks and hoods deform the wearer's voice, but clear communication is still possible in relative quiet over short distances. Some masks have a built-in vocal membrane to facilitate conversation.

- Mask tightness - it is very important to ensure a close fit to the wearer's face. Masks and half-masks are available in different sizes, so it is important that wearer's carefully check the tightness of their particular mask before entering the hazardous environment. For men with particularly thick facial hair, there are, for example, masks with extra airflow systems that produce some hypertension in the face (Fig. 16 and 17).

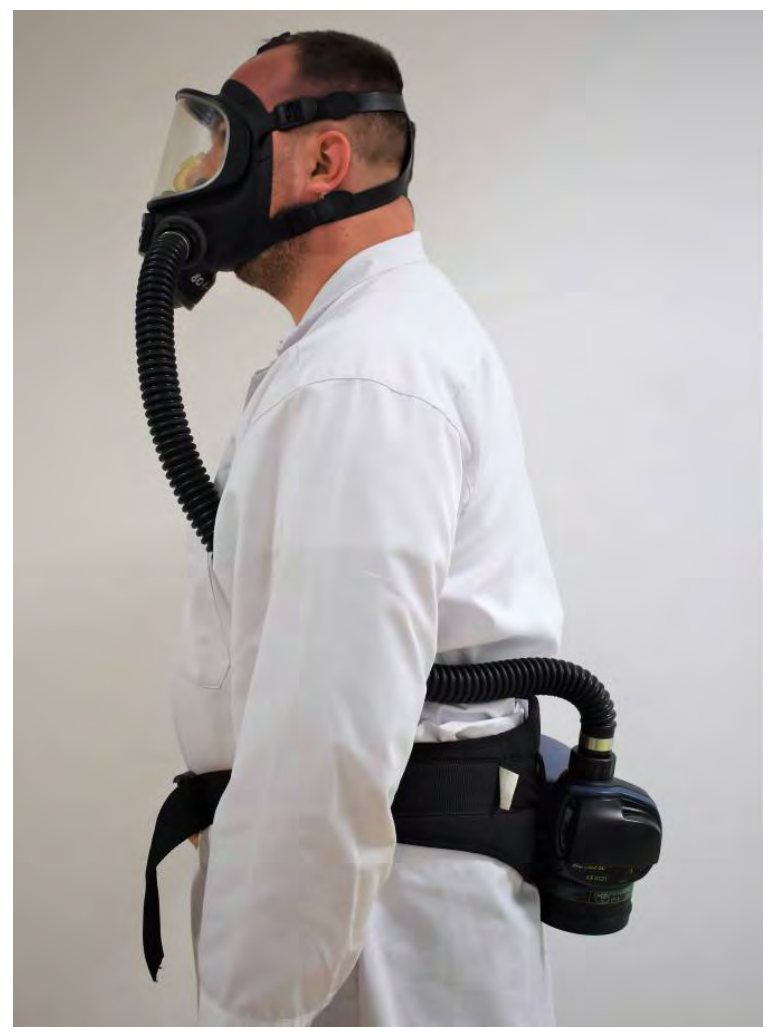

Figure 16. Mask with an extra airflow system (author's photo) 


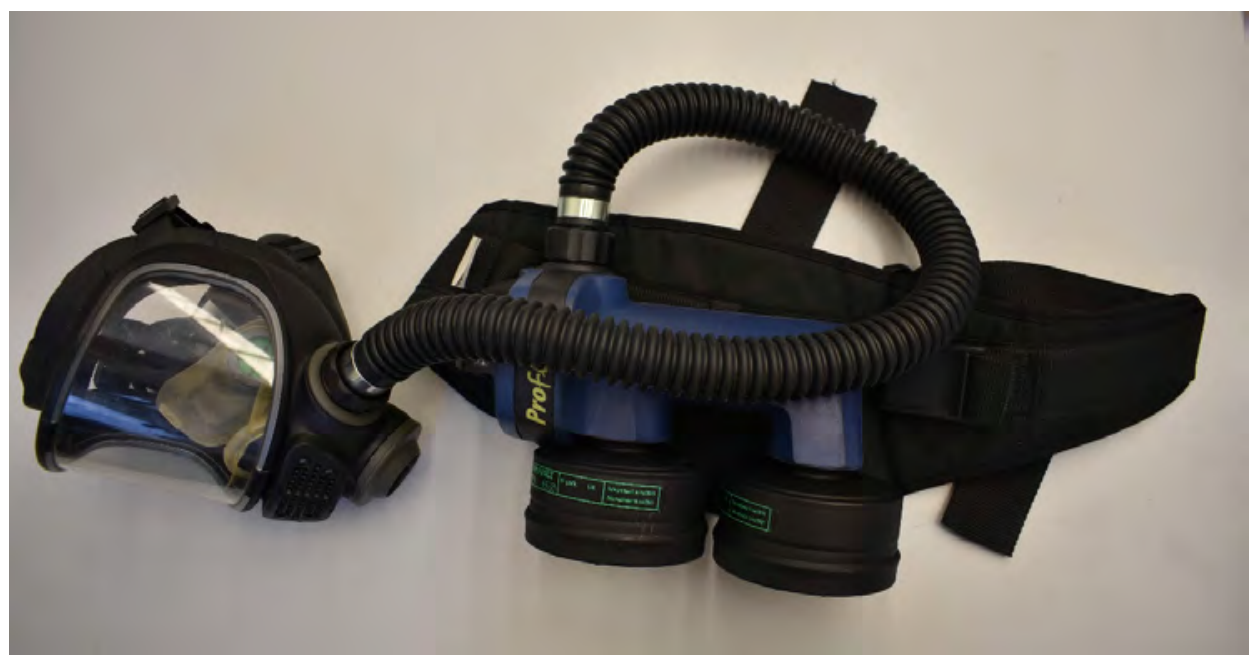

Figure 17. Mask with an extra airflow system (author's photo)

\subsection{Practical exercises in the use of personal protection equipment}

Practical exercises in the use of personal protection equipment include:

- Testing of and familiarisation with personal respiratory and skin protection equipment;

- Selecting the appropriate size mask, filter type and protective clothing;

- Correctly fitting masks and testing for leaks;

- Practising putting on protective equipment safely and rapidly (for emergency situations).

\subsubsection{Selecting the appropriate size mask, filter type and protective clothing}

For masks:

1. Measure the height of the face (from the bridge of the nose to the base of the chin) and use the result to select a mask based on the manufacturer's suggested sizes.

2. Check the surface of the mask for mechanical damage.

3. Surfaces in contact with the skin should be disinfected.

4. Install a new, suitable filter, an absorber or combined filter for the mask after identifying the type of hazardous substance involved (based on type and class (see Section 1, above). 
5. Always make sure that absorbers have been tested and approved for use with the hazardous substance involved.

6. Put the mask on, so that no hair comes between the mask and the skin.

7. Adjust the mask so that its adheres to the contours of the face.

For clothing:

1. Choose a size of suit appropriate for the wearer's height and chest circumference.

2. In the case of suits made of several elements, make sure that they are securely connected (Velcro, zip fasteners, elastic bands etc.).

3. Check that the hood is properly attached to the face part of the gas mask.

\subsubsection{Correctly fitting masks and testing for leaks}

To check the fit of a mask, use a PORTACOUNT device. This measures the number of naturally-occurring particles in the air and the number of particles under the mask (after passing through the filter/combined filter etc.

- Attach a mask of the correct size;

- Connect one of the filter ports on the filter or combined filter casing to the air sampler tube (Fig. 18);

- Have the user breathe calmly and normally for a minute;

- The PORTACOUNT unit simultaneously counts the number of particles in the air around the mask, and under the mask. It calculates the ratio of surrounding particles to the number of particles under the mask, giving the FIT FACTOR, a properly fitting mask will have a FIT FACTOR of at least 10,000.

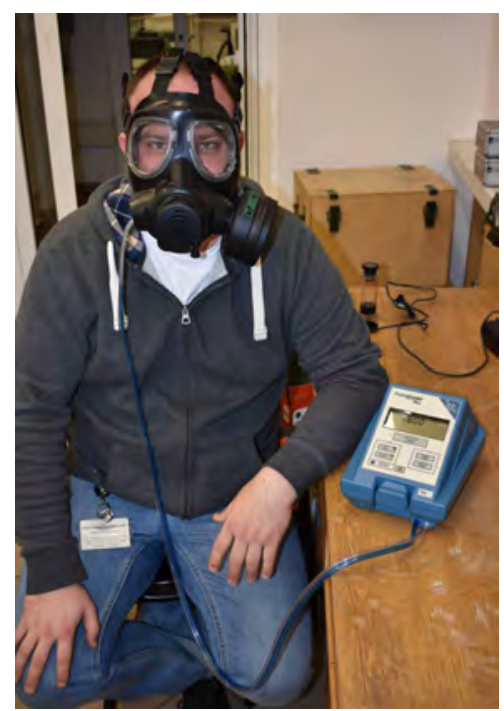

Figure 18. Checking the fit of a mask, using a PORTACOUNT (author's photo) 
Using a PORTACOUNT device, you can quickly determine if a mask fits the wearer's face properly by checking total internal leakage from the mask. The mask's user is located on a treadmill in a sealed chamber (Fig. 19 and 20). The interior of the chamber is filled with a sodium chloride aerosol. The user then performs a series of exercises to simulate the use of the mask in real-life conditions (fast walking, turning their head up and down and side-to-side etc.). Using a spectrophotometer, the concentration of sodium chloride in the chamber and under the mask is determined and the internal leakage is thus calculated.

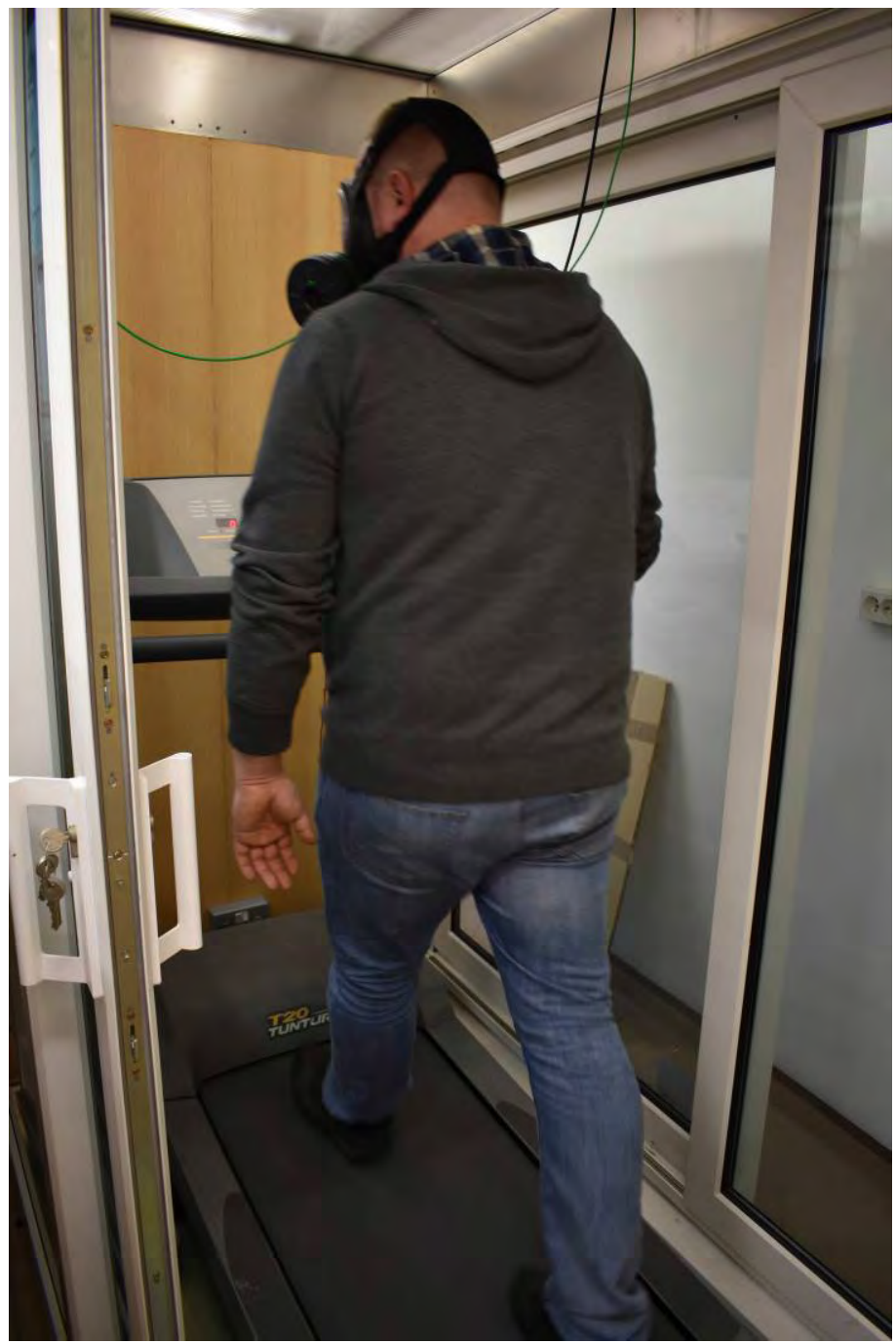

Figure 19. Checking total internal leakage (author's photo) 


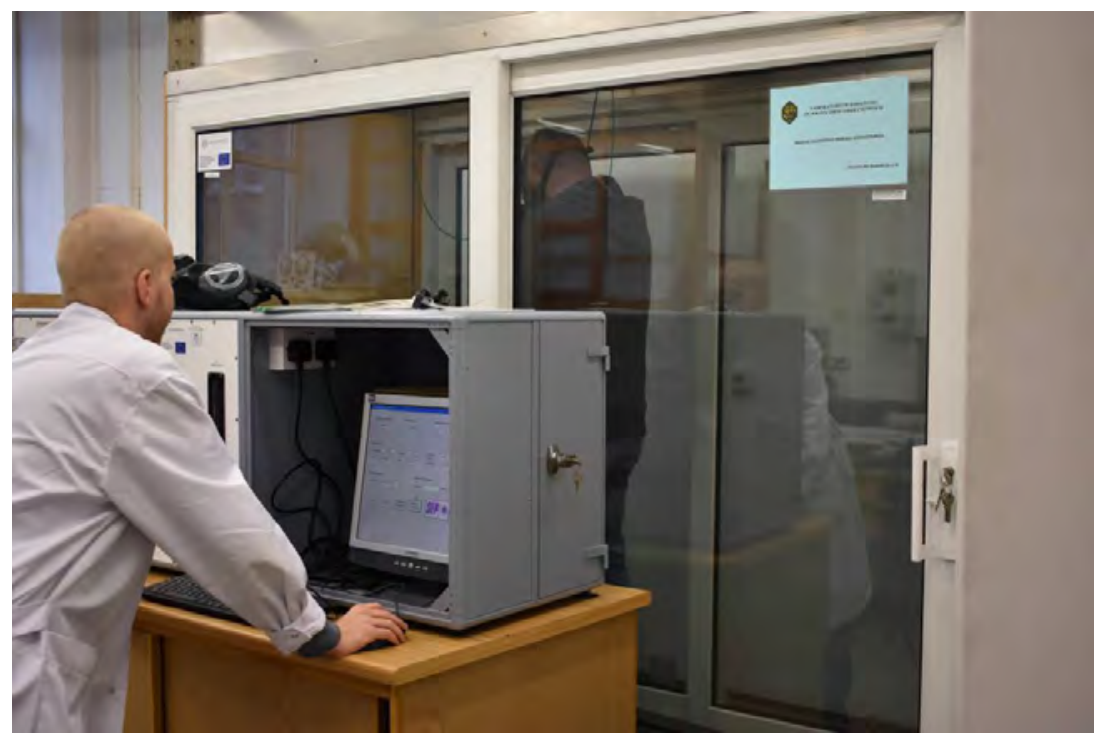

Figure 20. Checking total internal leakage (author's photo)

\subsubsection{Practising putting on protective equipment safely and rapidly}

In case of a rapid threat of contamination, operators should don the appropriate masks as soon as possible. The ideal time to correctly attach a mask is max. 9 seconds. After the filter's lifetime has expired, it must be replaced with a new one - keeping in mind that there may still be hazardous substances in the environment! To replace a mask filter in a hazardous environment:

1. Take a deep breath;

2. Hold that breath;

3. Unscrew the filter casing while beginning to very slowly and continuously exhale;

4. Insert the new filter and re-attach the filter casing;

5. Inhale. Remember, do not inhale without a properly installed filter!

\section{References}

European Standard EN 136.

Janes' Nuclear, Biological and Chemical Defence, Editet by John Eldrige, (19 ed.), 2006-2009.

Stanag 2352 - Nuclear, Biological and Chemical (NBC) Defence Equipment, 2013.

Wartell et al., Strategies to protect the health of deployed U.S. forces: Force protection and decontamination, National Academy Press Washington 1999. 